\title{
Publicar revistas de arqueología: cartografía académica y retos de futuro ${ }^{1}$
}

\author{
Publishing archaeological journals: \\ academic cartography and future challenges
}

Este ensayo intenta ofrecer unas reflexiones sobre las revistas especializadas de arqueología, articuladas en torno a las siguientes cuestiones centrales: primero, un análisis de la presión académica para publicar a toda costa y de los resultados negativos que puede llegar a ocasionar; segundo, dibujar una cartografía de las revistas especializadas de arqueología con referencias a su tradición historiográfica y tercero, realizar una valoración crítica del ecosistema cambiante de las revistas de las últimas décadas para comprender mejor la situación actual.

Por último, se intenta esbozar los principales retos de un futuro que ya ha llegado y que inevitablemente tienen que abordar todas las revistas: el sistema de evaluación de originales por pares, la defensa del valor intrínseco de los artículos, la decisión de la/s lengua/s en que publicar considerando sus implicaciones, el debate de la publicación en papel versus publicación electrónica y finalmente lo que significa hoy el prestigio académico, la descubribilidad y la visibilidad de las publicaciones periódicas.

Palabras clave: revistas de arqueología, publicación, gestión de publicaciones periódicas, evaluación por pares, publicación electrónica, sistemas de edición futura.
This paper offers thoughts on specialised archaeological journals articulated along three central issues. Firstly, an analysis of the academic pressure to publish at all costs and its potential negative results; secondly, the drawing up of a cartography of the archaeological magazines regarding their histographic tradition; and thirdly, advance a critical assessment of the changing ecosystem of journals in recent decades so as to shed light on their current situation.

Finally, we attempt to outline the main challenges of a future that has already arrived and that inevitably has to be addressed by all journals: the standard "peer review" evaluation system, the defence of the intrinsic value of articles, the choice and the implications of the language or languages in which to publish, the debate on paper versus electronic publications, and finally the current meaning of academic prestige, discoverability and visibility of periodicals.

Keywords: archaeological journals, publishers, journal management, peer review, electronic publication, future edition systems.

especializadas, en los que he aprendido mucho de colegas y amigos, especialmente de los de la Universitat de Lleida, un grupo admirable en muchos aspectos. Ellos son también, en última instancia, los responsables de este texto, que se ha beneficiado además de la estimulante Jornada de Celebración de los veinticinco años de $R A P$, que resultó muy interesante e inspiradora para los temas que aquí trato.
Este ensayo es deudor, en primer lugar, de largas y oscu británica de Southampton, en las que mi curiosidad exploratoria de la biblioteca desbordó las secciones de Prehistoria y Arqueología para descubrir y leer ávidamente revistas de educación, de escritura académica y del mundo de la edición. Interés que he mantenido desde entonces. Y en segundo lugar, de años de publicación, dirección y colaboración con revistas 


\section{Introducción}

Investigar es publicar. La publicación es la contribución más perdurable que cualquier arqueólogo puede hacer a su disciplina y a la sociedad (Connah 2010: 1). Además es una obligación profesional y ética de cualquier excavador (Lavell 1981), porque según el dictum académico publica o sé maldito sencillamente sin la publicación un arqueólogo destruye el registro como un saqueador de sitios arqueológicos. Excavar no es un experimento de laboratorio que pueda ser repetido cuando se quiera, ni como en la historia se puede volver sobre las fuentes, ya que han sido desplazadas en el proceso de excavación, deslocalizadas y descontextualizadas (Lucas 2001: 40). Solo la publicación restituye, en gran medida, lo destruido en la excavación arqueológica (VV.AA. 1986).

$\mathrm{Y}$ como en todas las disciplinas las publicaciones periódicas, las revistas especializadas, constituyen la avanzadilla de la investigación, el "frente de batalla" del conocimiento. Las tendencias, enfoques y estrategias más recientes - además obviamente de la información histórica- se encuentran en las revistas. Sin un conocimiento actualizado de lo que se publica en las revistas no puede haber buena investigación (Aubin 2012). Su significación es grande, en la medida en que aparecen con periodicidad cada vez más corta y permiten comunicar con rapidez a toda la comunidad arqueológica. Lejos de considerarse como simples receptáculos de nuevos conocimientos su valor va mucho más allá, porque juegan un papel muy importante y activo en la configuración de la propia investigación arqueológica (Rundkvist 2007) y en la formación académica de los futuros arqueólogos. Y al mismo tiempo, el desarrollo de nuevos subcampos de investigación origina nuevas revistas que se convierten así en testimonio de la relevancia de esas nuevas líneas investigadoras.

Por otra parte las revistas de arqueología son una representación de la comunidad científica que las produce $\mathrm{y}$, en última instancia, de las instituciones que se las apropian. De ahí que el estudio, en perspectiva historiográfica, de su vida constituya una aproximación fundamental para entender los perfiles de la investigación arqueológica, el establecimiento de cánones académicos y la naturaleza de las instituciones que las acogieron (Chippindale 2005). Ese es otro valor interesantísimo de las publicaciones periódicas (Gero y Root 1990; Mora 2002).

Hasta finales del siglo xx las revistas especializadas de arqueología se movían en un terreno firme que venía de lejos - las primeras revistas europeas aparecieron en los años 1830/1840 - y compartían unos principios cuasi-santificados: individualidad, originalidad, objetividad y propiedad intelectual. Operaban en un sistema de conocimiento que defendía la fijación, la uniformidad y los derechos de propiedad intelectual sobre los textos publicados (Pound 2013). La irrupción de la publicación electrónica en el mundo académico (Carver 2007a; Samida 2006; Waltham 2009) ha hecho tambalear estos principios, obligando, como acertadamente señala Pound (2013), a repensar estas asunciones. En las dos últimas décadas, con algunos indicios anteriores, está cambiando el carácter del conocimiento publicado, los propios soportes de publicación, el perfil de la investigación arqueológica, el concepto de autoría, la autoridad emanada de lo escrito en papel o la forma en que opera el sistema de evaluación por pares (peer-review) de los originales propuestos a las revistas (Gaillet y Guglielmo 2014; Richardson 2014).

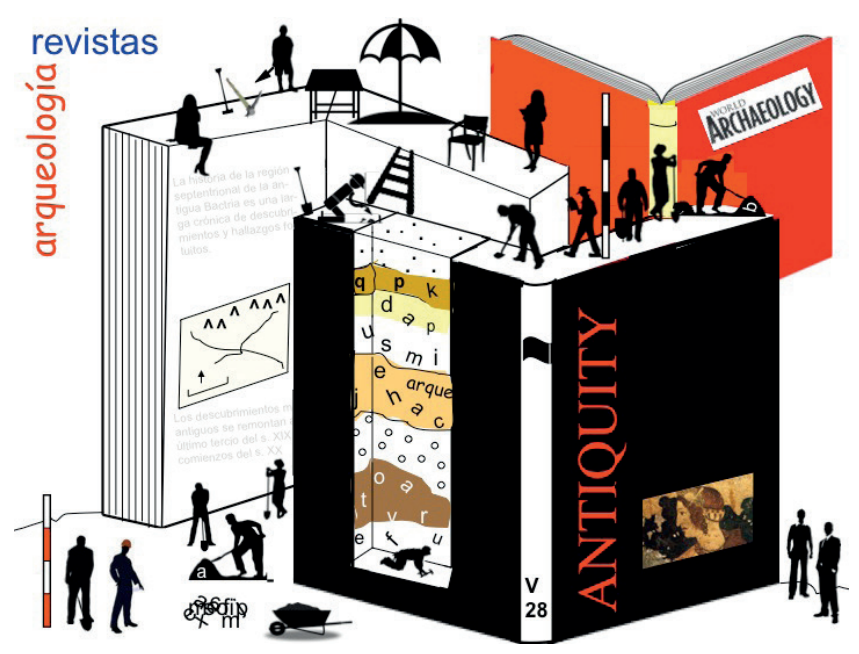

Figura 1. Cualquier iniciativa de mejora de las revistas de arqueología precisa de un buen conocimiento de su paisaje, estudio comparativo y ecosistema institucional. Todo ello pasa, de alguna manera, por excavar papeles considerando las propias revistas como artefactos arqueológicos.

Claramente hoy "todos somos arqueólogos digitales" (Morgan y Eve 2012: 523) y aunque la afirmación pueda parecer algo excesiva es una simple constatación de la práctica arqueológica de los últimos veinte años. Es cierto que "la arqueología digital está aquí y no se va a ir" (Costopoulos 2016). Pero otra cosa es que la edición electrónica de revistas especializadas esté asentada (Ames 2014), ya que las revistas exclusivamente electrónicas - pensadas desde lo digital y no como mera traslación del papel- son pocas, no se encuentran entre las mejores y no se aprecia un crecimiento significativo desde la aparición de la primera, Internet Archaeology, en 1996 (Xia 2006; Sideris 2004). Por más que sea completamente cierto que las buenas revistas de arqueología en papel no se conciben ya sin versión electrónica (Szuter 2014). Como veremos, el tránsito a la publicación electrónica exclusiva es uno de los grandes retos de la arqueología del siglo xxi y vale perfectamente esta lúcida reflexión para adelantar la consideración de una cuestión fundamental que abordaré más adelante: “Cambiar nuestras tecnologías, nuestras maneras de hacer investigación y nuestros modos de producir y distribuir los resultados de investigación son todos cruciales para la continuidad de la vitalidad de la academia; y ninguno de estos cambios puede llegar a menos que haya un profundo cambio en las formas en que los académicos piensan su trabajo. Hasta que los investigadores piensen realmente que publicar en la Web es tan valioso como publicar en papel... pocos [arqueólogos] estarán dispuestos a arriesgar sus 
carreras en una nueva manera de trabajar" (Fitzpatrick 2011: 10).

En ese sentido, una cosa es aceptar las numerosas ventajas del entorno digital y otra es el colonialismo digital que pretende que todo gire exclusivamente en ese entorno. Un análisis ponderado de los peligros del colonialismo digital, la defensa de la lectura en papel y una llamada a resistir contra la transición obligatoria a lo digital se encuentra en el excelente libro del filósofo italiano Roberto Casati (2015) Elogio del papel. Y es que cada vez contamos con más evidencias de que Internet no solo está creando nuevas formas de leer, procesar información y escribir sino que es muy posible que también lo esté haciendo en nuestra forma de pensar. En relación con las revistas de arqueología quiero enfatizar aquí las ventajas de la lectura en papel señaladas por Casati: 1) lectura de inmersión total controlada por el lector, 2) lectura sosegada y tranquila sin distracciones de los dispositivos digitales, y como consecuencia 3) lectura más atenta que permite la comprensión de textos largos y entrena para lecturas extensas y profundas. Algo que los dispositivos digitales han ido erosionando y comprobamos, sin ninguna duda, con nuestros alumnos quienes llevamos más de veinte o treinta años de enseñanza universitaria. Y eso no significa rechazar las muchas ventajas de lo digital.

Por último, un asunto fundamental para la edición exclusivamente electrónica y que no he conseguido que nadie responda afirmativamente. ¿Se puede garantizar que el formato de una revista electrónica será actualizado automáticamente cuando quede obsoleto? La arqueología no es un sector que genere beneficios económicos y por tanto, ¿quién empleará tiempo y dinero para la actualización de los formatos digitales? Las nuevas bibliotecas de investigación con repositorios digitales bastante tendrán con estar al día pero esa actualización se me antoja complicada, salvo que futuros desarrollos nos doten de entornos digitales tipo IKEA con la filosofía "hazlo tú mismo".

En este ensayo quiero considerar varias cuestiones: primero, analizar la actual presión académica para publicar y realizar una aproximación a la cartografía de las revistas; segundo, elaborar un bosquejo de la tradición de revistas especializadas en la disciplina; tercero, escrutar críticamente el ecosistema cambiante de las revistas de arqueología en las últimas décadas, para teniendo en cuenta todo lo anterior plantear, por último, los que a mi juicio son los grandes retos de futuro de las publicaciones periódicas de arqueología.

\section{Publica o perece o ¿publica y perece?}

La academia ha impuesto en las últimas décadas una presión, que crece fuerte y continuamente (Sarewitz 2016), porque el valor de sus miembros en cada especialidad se mide por el número e impacto de sus publicaciones. En ciencias duras eso significa el poder absoluto de los papers y su impacto medido por una cienciometría - scienciometrics y bibliometrics - que surgida a finales de los años 1970 (Price 1978; Nicolas y Ritchie 1978) ha ido haciéndose cada vez más sofisticada: los artículos valen lo que vale su factor de impacto. A mayor número de artículos y de mayor índice de impacto mayor valía del autor-investigador. Y aunque en Ciencias Sociales -y todavía más en Humanidades - los libros y monografías tienen un gran valor, los sistemas bibliométricos de las ciencias duras se imponen para todos y también para la arqueología. El mandato es bien conocido: "publica o perece”. El índice h de Hirsch (2005) cuantifica numéricamente la producción científica de un autor y su aparente impacto científico. Aunque resulta evidente que el número de citas de los artículos, por sí mismo, no hace relevante científicamente un artículo (Werner 2015) y que todos los indicadores cuantitativos deberían apoyar, nunca suplantar, el juicio crítico y razonado de un especialista (Wilsdon 2015).

La presión es efectiva sobre todo para la obtención de becas y plazas universitarias o de otros organismos de investigación por parte de investigadores jóvenes. Hay que buscar las revistas de más impacto para intentar publicar en ellas. En el caso de equipos potentes de investigación eso significa el diseño sofisticado y permanente de una auténtica ingeniería de publicación para promocionar a los miembros jóvenes y mantener a los experimentados con plaza fija. Según las coordenadas de la financiación de la moderna investigación si los investigadores pueden publicar más artículos simplemente lo harán (Arns 2014). Aunque en el caso español de algunos investigadores seniors la presión "normalizada" puede llegar a producir un efecto perverso: si para el sexenio de investigación universitario se necesitan cinco buenos papers algunos producen cinco artículos y nada más. Para qué más si con eso se tiene asegurado el sexenio.

Resulta evidente que con el crecimiento de los cultivadores de un campo científico en ascenso -y la arqueología sin duda alguna lo es- crece el número de revistas porque la producción de artículos se multiplica. Eso genera dos situaciones nuevas. Por un lado, el crecimiento de revistas ha creado nuevos nichos, es decir aparecen revistas punteras pero también otras grises, ya que por algún lado hay que empezar a publicar antes de llegar a las prestigiosas. En otras palabras, se amplía el espectro de las revistas. Por otro lado, el crecimiento de publicaciones es tal que resulta prácticamente inabarcable; para tener una mínima idea, solo la base de datos de Elsevier Scopus, uno de los mayores grupos editoriales académicos, revela que el número anual de artículos indizados subió de 1,2 millones en el año 2000 a unos 2,7 millones en 2013, lo que representa un $113 \%$ de crecimiento (Arns 2014). Y aunque lógicamente las cifras serían mucho más bajas si se midiera solo la arqueología, el volumen seguiría siendo muy grande y el crecimiento relativo también. A veces ya ni los abstracts, de mala calidad, permiten tener una idea clara de los contenidos (Bruce 2008). Un estudio amplio, pero centrado sobre todo en el ámbito anglosajón (Rocks-Macqueen 2015a: 44 ss.), revela que en la década de 1970 se crearon 33 revistas nuevas de arqueología, en la de 1980 fueron 35 y en la de 1990 llegaron a ser 43. Los valores de las españolas, con registros mucho más modestos, siguen esa tendencia general alcista.

Si la obsesión por publicar se dispara, los controles de calidad -básicamente la evaluación de originales por pares - se pueden relajar y paradójicamente el 
nivel de calidad de lo publicado descender. Algo que el sistema de revistas en Open Access puede acentuar. Si como sucede en el caso de la arqueología española el número de revistas y de manuscritos producidos crece y el número de investigadores con plaza no lo hace la presión sobre los investigadores instalados para actuar como evaluadores aumentará, se buscarán algunos no expertos en áreas concretas y puede decrecer la calidad de los artículos. La extraordinaria abundancia de información es ya agobiante y la posibilidad de contar con mecanismos que puedan filtrar bien los originales es cada vez más difícil.

La agobiante presión académica para publicar ha llegado al extremo de transmitirse a los estudiantes de arqueología. Eso, en gran medida, es lo que explica la aparición de revistas de estudiantes e investigadores jóvenes. Una iniciativa pionera fue la Archaeological Review from Cambridge fundada en 1981 (http://www.societies.cam.ac.uk/arc/home.html). En España la primera fue Arqueoweb (http://pendientedemigracion.ucm.es/info/arqueoweb/) creada en 1998 en la Universidad Complutense, a la que han seguido Estrat Crític (http://www.raco.cat/index.php/ EstratCritic) de la Universitat Autònoma de Barcelona en 2007, Arkeogazte (http://www.arkeogazte.org/) de la Universidad del País Vasco en 2011, y ArqueoUca (https://revistaarqueouca.wordpress.com/) de la asociación estudiantil Unión Cultural Arqueológica en la Facultad de Geografía e Historia de la Universidad Complutense que vio la luz ese mismo año. Y no se trata de criticar estas iniciativas, muy al contrario, vaya por delante que todas estas revistas resultan no solo de calidad sino también singulares, en algunos casos muy valiosas, en la medida que han publicado estudios sobre aspectos comprometidos y no convencionales que difícilmente encuentran lugar en otras revistas académicas. Simplemente es constatar como se transmite el valor de la publicación en investigación. En EE.UU. desde una revista de la prestigiosa Society for American Archaeology (Frame y Duwe 2009) se han proporcionado útiles consejos para que los undergraduated fueran preparando su camino en la publicación, orientando sobre: conciencia de su significado e importancia, búsqueda de opiniones contrastadas, por donde empezar a publicar, y como contactar con las revistas académicas. En 2016 se ha fundado el International Journal of Student Research in Archaeology (http://www.ijsra.org/), la primera revista de este género, independiente y con vocación internacional, dedicada a publicar investigación estudiantil de todo el mundo, promoviendo el diálogo y la inclusividad de la disciplina (Linares Matás 2016).

Ciertamente se está publicando mucho, aunque por descontado no todo es relevante, e incluso hay bastantes productos perfectamente prescindibles. Pero las propias plataformas académicas en línea del estilo de Academia.edu o ResearchGate nos animan a publicar más y más, a comunicar a colegas amigos pero también desconocidos nuestros trabajos subidos alentando la compulsión por publicar. Los datos bibliométricos de fuentes como Elsevier, Google Scholar y Thomson Reuters ISI comunican cuales son nuestros scores, e igualmente nos alientan a mejorar los índices de productividad, que presumiblemente siempre podrán ser mejores. Pocas voces se levantan para decir que este paisaje de la publicación masiva - y en algunos casos incontrolada- no puede traer nada bueno. ¿Publicar y perecer? ¿Es ese el camino en que estamos ya? ¿Cuál es la ratio entre publicaciones valiosas y publicaciones grises?

Desde luego la pregunta inquietante es: ¿Hemos alcanzado ya un nivel de saturación en el que la presión por publicar amenaza la buena publicación? El profesor Sarewitz, de la Universidad de Arizona, ha expresado recientemente su alarma sobre el peligro real de que la investigación relevante se vea sumergida por el creciente número de trabajos mediocres y sugiere que el crecimiento exponencial puede llevarnos a un escenario diferente, desconocido pero además amenazador (Sarewitz 2016). Propone que publicar menos y con menos frecuencia, a pesar de lo que nos prometan los correos electrónicos promocionales de plataformas y consorcios editoriales, puede ser la clave. Estoy convencido de que el consejo es prudente y realista pero ¿quién va a cambiar la maquinaria y la "lógica" de la burocracia académica? Solo la construcción de una trinchera académica que vaya sumando defensores contra la burocracia académica podrá lograrlo algún día: se necesitan valientes.

Ante la inundación de publicaciones se ha llegado a sugerir que podremos disponer de un buscador de revistas de arqueología como los de viajes, hoteles y restaurantes (Perkel 2015) y que podremos calificar a las revistas. De hecho ya existen grandes websites de comparación de revistas científicas y decenas de miles de títulos.

\section{La tradición de las revistas de arqueología}

Desde el inicio de la tradición de revistas de arqueología, a finales del primer tercio del siglo XIX en Europa (Archaeological Journal 1832, Archäologische Zeitung 1832 y Revue Archéologique 1844) y algo más tarde en España (Mora 1995), su número y complejidad no ha dejado de crecer hasta la actualidad. Aunque no contamos con ningún estudio exhaustivo de la evolución global de las revistas de arqueología es muy posible que los intentos parciales centrados en el ámbito anglosajón (Rocks-Macqueen 2015a, 2015b) permitan al menos detectar una evolución que probablemente no sería muy distinta si incluyéramos otras tradiciones arqueológicas (Aubin 2007). Según los datos recopilados por Rocks-Macqueen (figura 2) la secuencia sería aproximadamente así: en las últimas décadas del siglo XIX se crearon nuevas revistas; otro pequeño impulso se operó en el tiempo de entreguerras; un crecimiento más o menos continuado se produjo entre finales de 1940 y comienzos de 1980; la "década prodigiosa" fue, sin duda alguna, entre 1985 y 1995, y tras un crecimiento posterior más moderado parece que en los últimos años asistimos a un nuevo repunte en la aparición de nuevas revistas, ligado al surgimiento de nuevos subcampos disciplinares, la publicación electrónica y el Open Access (Canny 2015).

Esa evolución general es más interesante si se tiene en cuenta, como hace Rocks-Macqueen (2015a), 


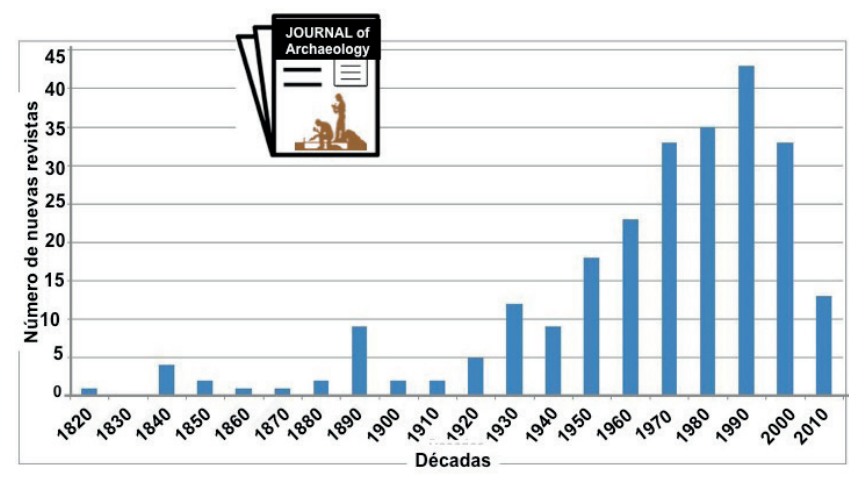

Figura 2. El crecimiento de las revistas de arqueología anglosajonas por décadas desde el siglo XIX al XXI (según Rocks-Macqueen 2015a y 2015b).

la evolución comparada en el último cuarto de siglo de los tres tipos básicos de revistas: 1) las institucionales, 2) las comerciales ligadas a grandes grupos editoriales científicos y 3) las incorporadas al sistema Open Access. El gráfico comparativo (figura 3) revela detalles y matices interesantes. Primero, el "gran salto" en la edición de revistas de arqueología se inicia a mediados de la década de 1970 con un crecimiento fuerte a partir de 1990. Segundo, las revistas institucionales son las que han tenido el crecimiento más moderado, con una ligera tendencia al alza en los últimos veinte años, mientras que las revistas comerciales han experimentado un crecimiento importante a lo largo de los últimos veinticinco años y finalmente las de Open Access son las que han experimentado el crecimiento más fuerte, especialmente desde 2003 (fecha de la declaración de Berlín), y esa tendencia presumiblemente se incrementará (Kohle 2015). El lado oscuro del Open Access es que la explosión de publicaciones ha alimentado el crecimiento de editores/ operadores cuestionables y a veces nada respetables (Butler 2013). La realidad es que, también en arqueología, el futuro de la publicación científica será en Open Access, especialmente si consideramos que la mayoría de los fondos de investigación son públicos (Harding 2015; Xia 2011).

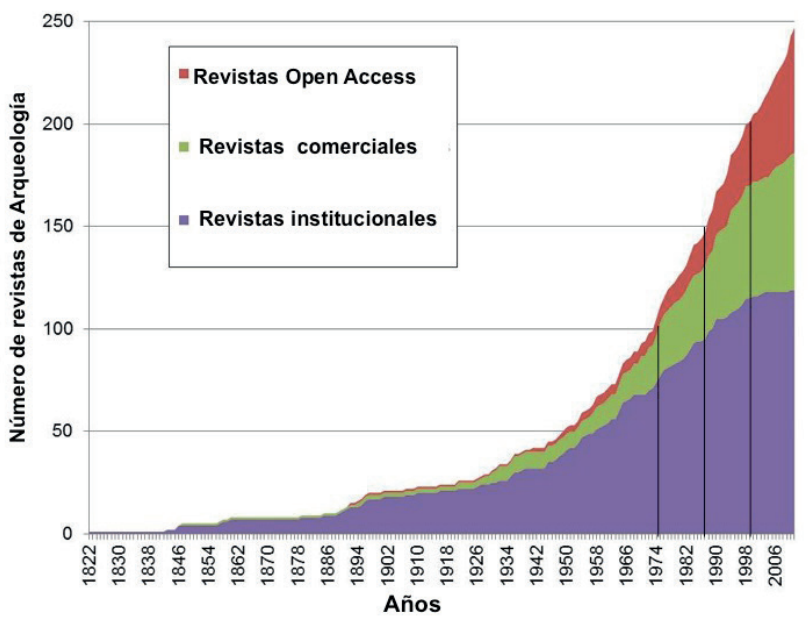

Figura 3. El crecimiento comparado de las revistas de arqueología anglosajonas por tipo de edición, desde el siglo XIX al XXI (según Rocks-Macqueen 2015a).
Pero tener otro punto de vista diferente al de la tradición arqueológica anglosajona es fundamental porque la arqueología mundial, hoy día, está fragmentada en bloques (Mizoguchi 2015: 19-20). En ese sentido se puede comparar lo anterior con un interesante estudio de las revistas de arqueología francesas. En el caso de Francia el extenso y detallado informe del Comité des Publications et de la Diffusion de la Recherche Archéologique (Aubin 2007) revela, en primer lugar, el fuerte tejido institucional de la arqueología francesa (un informe parecido en nuestro país se me antoja casi imposible, aunque véase para compensar el excelente ensayo de L. Armada en esta misma revista) y en segundo lugar la existencia de una red de revistas arqueológicas bastante organizada y bien jerarquizada (algo también alejado del sistema español), ya que las revistas se clasifican claramente en tres niveles: 1) regional, 2) interregional y 3) nacional. Por otra parte, el tejido editorial demuestra que existe una diversidad de actores - lo que previene del "pensamiento único"-, que esos diversos editores cohabitan razonablemente bien y desarrollan esfuerzos para racionalizar recursos y lograr objetivos, que las revistas están abiertas a recoger resultados de la arqueología preventiva aunque se abran interrogantes de cara al futuro, y que la caída de ventas/suscripciones, inevitable por varios factores, pero principalmente la nueva accesibilidad digital, se ha traducido en la reducción de la tiradas de papel (Aubin 2007: 133). De manera que se concluye afirmando que la edición arqueológica constituye una suerte de biotopo que tiene una organización jerarquizada —los tres niveles señalados—, y además complementaria, a la hora de dar a conocer nuevos conocimientos y hallazgos (Aubin 2007: 154). En cuanto al crecimiento de revistas parece que el modelo francés es bastante estable, y en la situación actual incluso se preconiza que antes de crear nuevas revistas se mejoren las ya existentes (Aubin 2007: 171). La diversidad de editores es muy importante y el modelo francés sigue la tendencia internacional de las revistas de humanidades que resisten bien al oligopolio de los grandes grupos editoriales (Lavivière et al. 2015).

Y estoy muy de acuerdo en lo que defiende el informe sobre una revista especializada cuando afirma que "una revista no es solo el receptáculo de artículos en los que se puede confiar" sino que debe ser "un actor de la vida arqueológica" que se oriente con voluntad firme hacia objetivos claramente definidos (Aubin 2007: 156) porque solo así producirá los mejores resultados. Yo lo llamaría la personalidad de una revista, que lógicamente se puede tener, con distintos grados, o simplemente carecer de ella. Personalidad que también se puede adquirir si los gestores de la revista son facilitadores de propuestas de investigadores líderes que son los que marcan el camino (Torrence et al. 2015: 7).

En el caso español aquí solo pretendo dar una mínima pincelada de la situación de las revistas de arqueología (Mora y Ramírez en prensa). La tradición española mantiene pocas cabeceras de antes de 1950, lo que ya traduce de alguna forma la poca entidad del tejido editorial y la inconstancia del existente (Ruiz Zapatero y Álvarez Sanchís 1989). En cualquier caso 


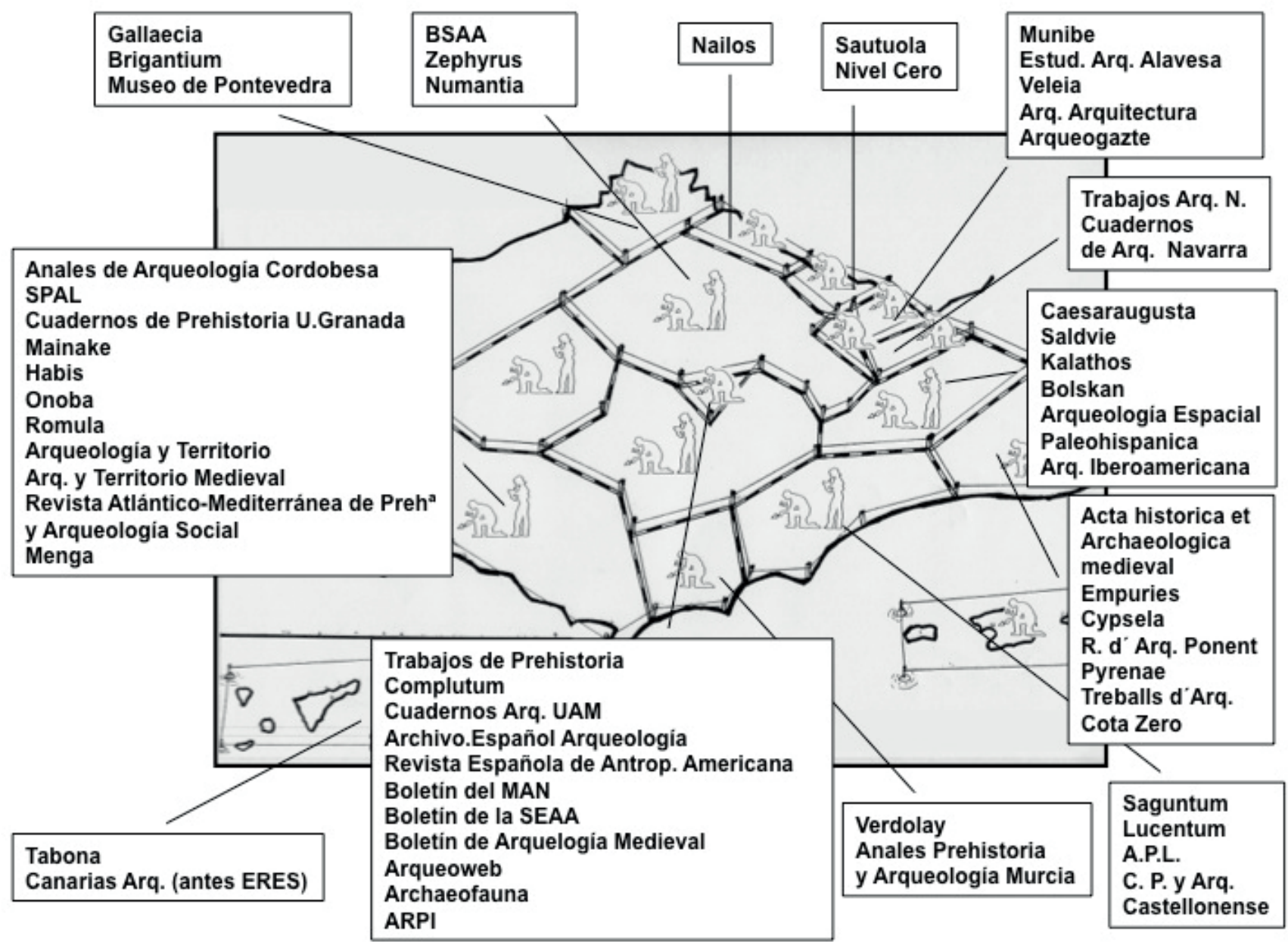

Figura 4. Aproximación a una cartografía de las revistas de arqueología por Comunidades Autónomas. Un escenario cambiante en el que se han omitido las revistas más abiertamente de numismática y epigrafía. (Para una visión muy completa ver Armada en este mismo volumen.)

son algo más de sesenta las revistas vivas, aunque la manera de medir esa condición en nuestro país sea compleja por los retrasos de aparición (figura 4). En la década de 1970 se produce la fundación más importante de nuevas revistas y luego habrá otro repunte en los años 1990. En los últimos años han seguido apareciendo nuevas cabeceras, algunas exclusivamente electrónicas, pero también han ido desapareciendo otras. La inmensa mayoría son institucionales y Andalucía, Cataluña y Madrid editan la mayor parte de revistas (Ver Armada en este mismo volumen). Como retos de futuro planteaban Mora y Ramírez (en prensa) los siguientes: primero, sobrevivir que está resultando complicado en bastantes casos; segundo, cumplir la periodicidad porque sin ella no se pueden abordar otros objetivos; tercero, mejorar la gestión editorial para tener revistas atractivas para los investigadores; cuarto, crecer en visibilidad, fundamentalmente en bases de datos y repositorios (McManamon y Kintigh 2010) y finalmente publicar en abierto digitalmente para llegar más lejos y a más audiencias.

Sobre el posicionamiento del nivel de calidad de las revistas españolas de arqueología hay que subrayar que la estructura del estado de las autonomías ha fragmentado los estándares de clasificación de las mismas (Martínez Navarrete y Montero 2004), una situación ciertamente no deseable.

\section{Repensando el ecosistema de las revistas de arqueología en un mundo cambiante}

El número de revistas de arqueología, globalmente, es muy grande y existen algunas cuestiones que tienen que ver con su futuro. Aquí me voy a centrar en presentar algunas reflexiones sobre los formatos y contenidos de las revistas y sobre la importancia de producir revistas con personalidad propia.

Sobre los formatos/contenidos, se debe destacar en primer lugar cuales son los tipos de trabajos que incorporan las revistas. Los más numerosos son obviamente los artículos y notas, habitualmente diferenciados por su extensión. Muchas revistas solo cuentan con estos dos tipos de contenidos, sencillamente porque es lo que resulta más fácil. A esto pueden añadir excepcionalmente las necrológicas. Hay contenidos que exigen infraestructuras importantes, sobre todo de equipo humano, por ejemplo contar con una sección fija de reseñas (Ruiz Zapatero 1987, 
1997) y/o artículos-recensión (comentario conjunto de varios libros con afinidad temática), críticas de exposiciones, películas o performances, y tener un editorial en cada número, no solo excepcionalmente por el cambio de dirección o editor. Algo parecido se puede decir para incluir dosieres, varios artículos de distintos autores sobre un tema propuesto por la revista o el estilo de artículo de Current Anthropology, un texto de encargo a un especialista que se somete a comentarios de varios investigadores y una réplica final del primero. En otros casos la consideración tradicional de que los contenidos se deben limitar a publicar artículos convencionales excluye contenidos interesantes como las entrevistas a investigadores notables, muy escasas en general pero que ofrecen gran interés si se seleccionan bien autores y se prepara un buen cuestionario. Suelen ser también escasas las revistas que incluyen listados de nuevos libros y monografías. Que los tipos de contenidos pueden ser más heterodoxos lo demuestran los ensayos fotográficos o artículos-en-formato-cómic (Swogger 2015), aunque por ahora solo sean experiencias casi únicas.

La publicación simultánea papel-digital está permitiendo, como hace Antiquity, alojar contenidos - por ejemplo los obituarios- solo en la versión digital para no cargar demasiado la edición en papel. Flexibilidad que puede proporcionar interesantes posibilidades.
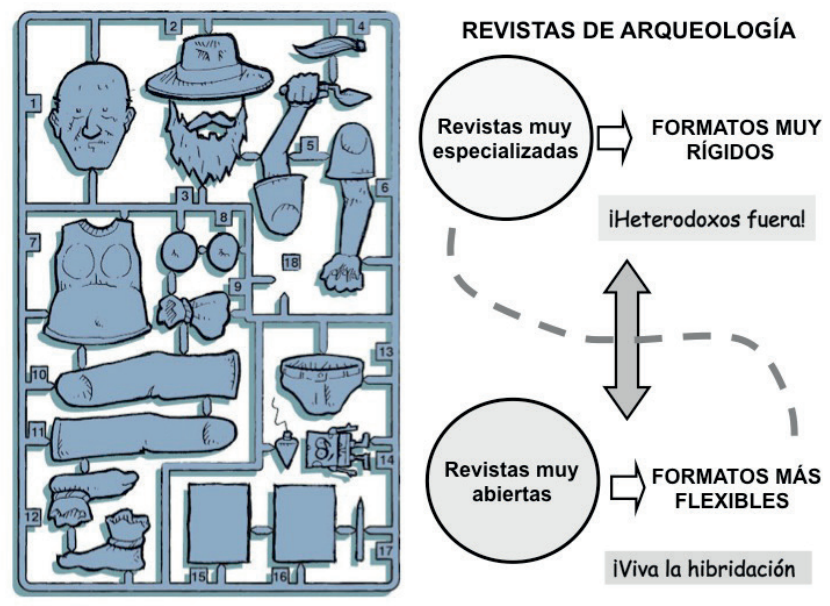

Figura 5. Gradación de los formatos de las revistas de arqueología. (Ilustración de McHale, $<$ http://conormchale.blogspot.com.es/search?updated-min= 2011-01-01T00:00:00Z\&updated-max=2012-0101T00:00:00Z\&max-results $=50>$.)

Volviendo al tema de la personalidad de las revistas y con la opinión de que una revista de arqueología debería ser algo más que un receptáculo de artículos confiables creo que hay revistas con personalidad y otras sin ella. En primer lugar aunque sea simplificar en exceso hay revistas muy especializadas con formatos muy rígidos, especialmente en el caso de las revistas de arqueología "más científicas", caso del Journal of Archaeological Science y otras similares con un ámbito muy pegado a las ciencias duras, en las que es casi imposible no ajustarse exactamente al estilo y requerimientos del editor. En el otro extremo están
Transdisciplinaridad

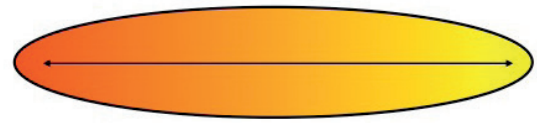

Interdisciplinaridad

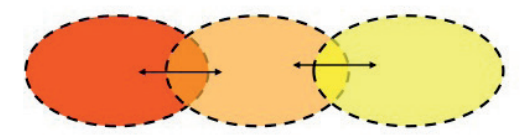

Multidisciplinaridad

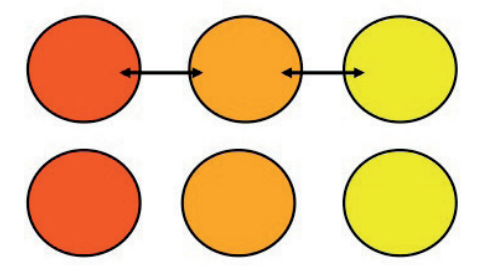

Disciplinaridad

Figura 6. Las revistas de arqueología se pueden clasificar por su orientación en: disciplinares, la gran mayoría, que tienden a reproducir su orientación por los evaluadores que emplean; multidisciplinares, acogiendo textos de diferentes disciplinas, algo relativamente habitual; interdisciplinares, mucho más escasas, cuando enfocan un mismo tema desde distintas disciplinas, y transdisciplinares, cuando los temas abordados diluyen las fronteras entre disciplinas tradicionales (según <http://www.nature.com/nchembio/journal/v4/n9/images/ nchembio0908-511-F1.jpg >).

las revistas muy abiertas, con formatos más flexibles y más predispuestas a cierta heterodoxia (figura 5). Entre unas y otras hay un campo amplio de gradación en el que se sitúan muchas otras revistas. Esa gradación tiene también que ver con la orientación, desde las estrictamente disciplinares, pasando por las multidisciplinares para llegar a las interdisciplinares y especialmente las transdisciplinares, las más valientes y transgresoras (figura 6). Un buen ejemplo de estas últimas es el Cambridge Archaeological Journal creado para el desarrollo transdisciplinar de una arqueología simbólica, social y cognitiva.

Para que una revista logre personalidad y éxito en su posicionamiento en el mercado académico no hay recetas mágicas pero al menos sí se pueden destacar algunos rasgos ganadores. Se precisa para ello: 1) singularidad, que significa perfil propio y diferenciado de la mayoría, adquiriendo así un valor único y específico; 2) rigor y calidad de los trabajos publicados, no hay nada más disuasorio para buenos investigadores que ver revistas con estándares pobres; 3) targets ambiciosos, es decir voluntad de dirigirse a audiencias del escalón más alto de investigación sin dejar de ser por ello accesibles y 4) operar con un nivel multiescalar, es decir ir de lo local y concreto a lo más global y general, sin resultar ni localista ni etéreo (figura 7).

$\mathrm{Y}$ aunque resulte algo absolutamente personal y por tanto subjetivo no me resisto a comentar algunos casos de lo que podríamos llamar revistas con personalidad y por tanto con un valor añadido. Antiquity ocuparía un lugar especial por su cobertura mundial, ritmo de publicación (seis fascículos anuales), singularidad de sus editoriales y secciones de recensiones y nuevas publicaciones, su Web Site y el Open Access Project Gallery. Los artículos con comentarios de Current Anthropology constituyen un género casi 


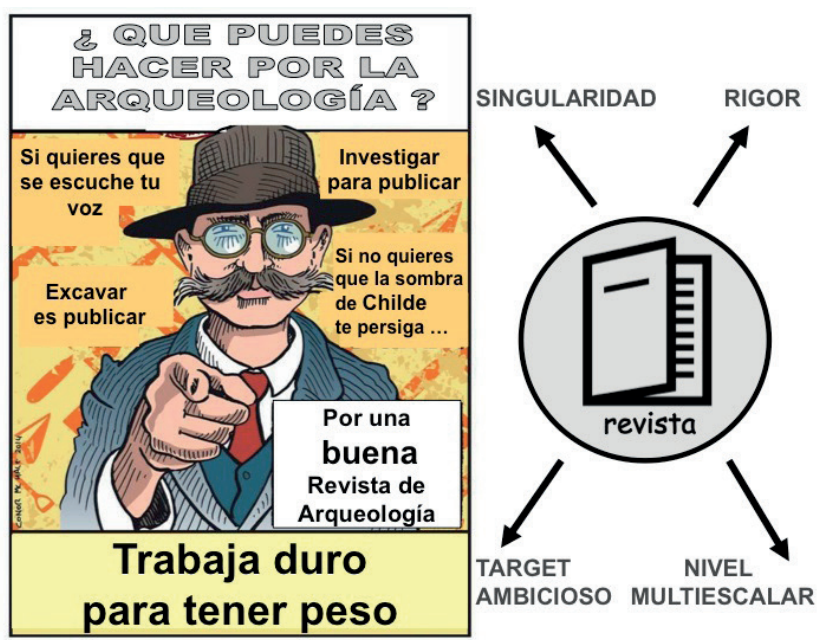

Figura 7. Rasgos más significativos para lograr una revista de arqueología competitiva. (Ilustración de C. McHale, modificada, <http://unitearch.wix.com/unitearchaeology\#!joinunite-now-(conor-mchale)/zoom/mainPage/i01o2w>.)

único, actualmente seguido por la interesante Archaeological Dialogues. Singular también por el carácter monográfico de cada número es World Archaeology. Por lo mismo, con dosieres muy buenos y en temas poco habituales, más una información muy completa de novedades, trabajos universitarios y exposiciones destaca Les Nouvelles de l'Archéologie aunque en este caso el ámbito no rebasa mucho el territorio francés. En el caso español he señalado ya las revistas "de estudiantes" con perfiles propios, novedosos y valientes. Por su parte, Trabajos de Prehistoria estableció y mantiene la mejor sección de recensiones, mientras que Revista d'Arqueologia de Ponent ha hecho lo propio con una sección de Debates abordando cuestiones interesantes y de actualidad que casi ninguna otra revista hace. Por su parte Complutum ha publicado dosieres y números extra con temas de interés y nivel verdaderamente internacional.

La apuesta de futuro para las revistas españolas pasará por ganar personalidad, mantener calidad y conseguir el mayor nivel de proyección internacional. El paisaje editorial cambiante exigirá también la búsqueda continua de fórmulas para atraer al mayor número de lectores, contar al mismo tiempo con alto rigor académico y, en definitiva, conseguir ser influyentes en el campo de la arqueología.

\section{Los retos de futuro ¿o más bien de ahora?}

Los cinco retos que se abordan a continuación, pensados para el presente y futuro inmediato, no pretenden agotar los retos que tienen ante sí las revistas académicas sino que simplemente son los que personalmente me parecen más importantes para la publicación en arqueología.

\section{La evaluación por pares}

La evaluación de originales mediante la evaluación de varios pares, investigadores cualificados en el tema del artículo, la peer-review, es hoy día el sistema menos malo para asegurar la mejor calidad en los artículos que se publican en una revista. A pesar de que la mayoría estamos convencidos de que esto es verdad, alrededor de la evaluación por pares han ido surgiendo quejas que apuntan a las limitaciones intrínsecas del procedimiento (Pinholster 2016), a usos malintencionados (Ferguson et al. 2014) y a sesgos por parte de los directores y/o consejos de redacción,
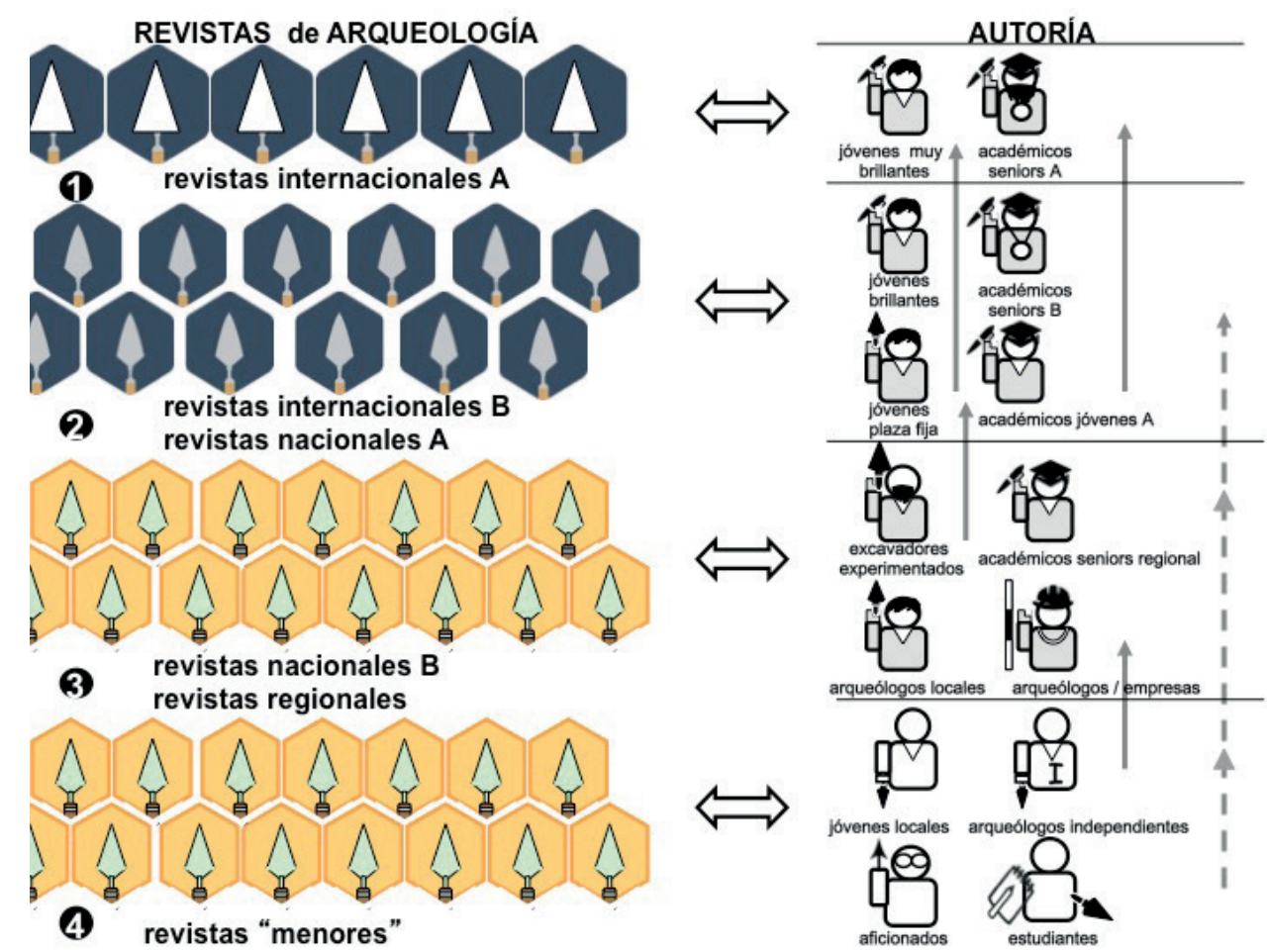

Figura 8. Modelo teórico de clasificación de revistas de arqueología y autoría potencial para cada tipo de revista. Un estudio exhaustivo de revistas y autoría proporcionaría resultados muy interesantes: es una cartografía por construir. 


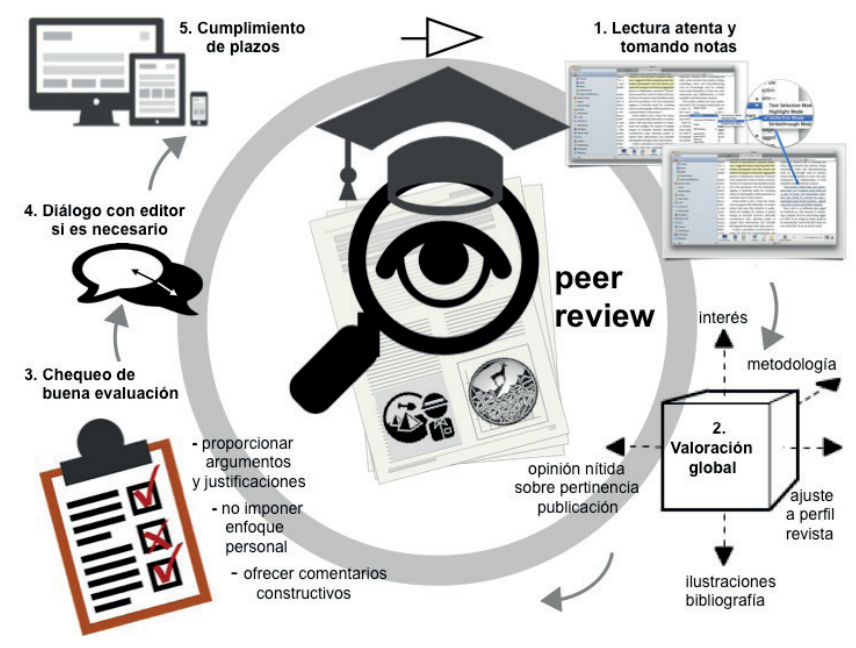

Figura 9. Ciclo del sistema de peer-review como proceso clave para el establecimiento de un alto nivel de calidad en una revista especializada.

especialmente en la elección de los expertos (Kotb 2016). Y debemos recordar que sigue habiendo muchas cosas que no conocemos del proceso integral de peer-review (Meruane et al. 2016) y que ayudarían a mejorarlo en la práctica cotidiana; como por ejemplo el análisis detallado de los textos intercambiados a lo largo del proceso de evaluación (Editor = Evaluadores $=$ Autor $/$ es $=$ Editor $)$. Un experimento valioso que refleja la doble actuación como evaluador y como autor para mostrar el proceso de peer-review "desde dentro" (Vora y Boellstorf 2012) resulta muy aleccionador. Y en cualquier caso, la obligación más básica de una revista de investigación es trabajar con el proceso de peer-review (Bohannon 2013: 65).

Se ha sugerido recientemente que el proceso de evaluación por pares, de alguna manera, tiene sus remotos orígenes en el siglo XVI durante la Reforma Protestante cuando se quemaban libros según las facciones religiosas, la Anglicana y la Católica (Lipscombe 2016). Para evitarlo se pedía a expertos una estampa de aprobación de los libros. Así "de las cenizas de los libros quemados surgió el ave Fénix de la peer-review" (Lipscombe 2016: 295).

Como he señalado más arriba, con todos los problemas que rodean a la peer-review -y ciertamente son bastantes - nadie ha demostrado un sistema más eficaz para valorar manuscritos y mantener una alta calidad de lo publicado. Y el sistema de peer-review de "doble-ciego" (se mantiene anonimato de autores y evaluadores simultáneamente) se considera el más eficaz (Announcement 2015). Cierto que en subcampos pequeños mantener el anonimato de autor/es cuando hay referencias inequívocas es casi imposible y algo parecido se puede decir con la presión de las instituciones para que las revistas publiquen la relación de evaluadores cada año (por tema y nombre el evaluado puede adivinar quienes han sido sus evaluadores). Esto último me parece que es un exceso y no acabo de comprender bien su finalidad. ¿Exhibir que se ha contado con reputados especialistas?

Aunque las revistas proporcionan generalmente formularios con campos que recogen los principales parámetros a evaluar, es cierto que no hay - hasta donde conozco- reflexiones profundas sobre las implicaciones y las formas de cómo realizar evaluaciones serias, constructivas y clarificadoras (pero véase: Donovan 2014; Lovejoy et al. 2011). Sí hay en arqueología orientaciones sobre como contestar las observaciones de los evaluadores (Connah 2010: 166171). Y sostengo que no es nada fácil redactar buenas evaluaciones. Algunos grupos editoriales, como el de Taylor\&Francis Group (2015), difunden orientaciones que pueden resultar útiles. Pero sin duda, además de algunas instrucciones formales, lo que resulta más valioso es la experiencia destilada de años sirviendo a la comunidad científica en esta tarea y gestionando las evaluaciones de revistas (Schiermeier 2016). Una consideración más detallada muestra como la evaluación de manuscritos es una obligación moral de los académicos, que deben dedicar toda su atención e interés en la tarea, porque es muy importante contribuir a elevar el nivel de la investigación de nuestra disciplina. Los buenos académicos se toman la evaluación de manuscritos muy en serio y ello forma parte integral de su reputación (Woolston 2015: 114). Porque piensan que toda evaluación de un manuscrito es, en primer lugar y antes de nada, un ejercicio de apreciación del texto considerado. Buenas evaluaciones construyen buenas revistas y garantizan la ausencia de errores sustantivos en lo que se publica. Y es que los evaluadores son, de alguna manera, los "héroes no-reconocidos" de las buenas revistas (Torrence et al. 2015: 6). A pesar de todo, los casos de retractación - se obliga a retirar un paper que ha pasado el filtro evaluador por denuncias de deshonestidad una vez publicado on line- surgen en la mejores revistas (Editorial 2015) y abren la puerta a un nuevo concepto la "evaluación post-publicación" por parte de toda la comunidad científica, perfectamente factible en la edición electrónica.

La gestión de las evaluaciones es compleja porque se deben elegir bien los evaluadores, buscando siempre la especialidad o en su defecto académicos con amplia trayectoria y buen sentido crítico. Si se eligen evaluadores muy jóvenes se corre el riesgo de que actúen con un acusado "integrismo" y falta de perspectiva amplia aunque pueden ser muy buenos en los detalles y conocimiento de los datos así como de métodos y aproximaciones más novedosas. Los evaluadores seniors pueden ser más difíciles de convencer para que acepten, por sus múltiples tareas, pero si tienen sensibilidad académica lo harán siempre que realmente puedan. De alguna forma suelen aportar valoraciones más equilibradas y con visiones más generales y flexibles. En última instancia los campos de investigación no acostumbran a ser muy grandes y como ha dicho Woolston (2015: 113) es casi como vivir en un pueblo: hagas lo que hagas no puedes huir de lo que se conoce de ti; si evalúas mal y con prisas al final eso trascenderá de alguna forma y tu reputación se verá afectada. Es verdad que muchos académicos de una disciplina conocen genéricamente quienes son buenos evaluadores y quienes no.

No siempre resulta fácil transmitir a los evaluadores que no se trata de decir a la revista lo que debe publicar y cómo sino de proporcionar argumentos 
sólidos y justificaciones detalladas de sus apreciaciones (Schiermeier 2016: 280) con la finalidad de que la revista tenga así criterios nítidos para tomar una decisión y pueda trasladar mensajes claros y constructivos a los autores para la revisión/mejora de sus textos.

\section{La defensa de textos originales}

La preocupación por las publicaciones mientras se desarrolla la tesis doctoral es una recomendación bastante generalizada, personalmente siempre lo he hecho con mis doctorandos y siempre insisto en que al leer una tesis doctoral se deberían tener unos pocos artículos ya publicados. De hecho cuando empiezo a leer una tesis lo primero que miro es la lista de bibliografía para descubrir las entradas que tiene en ella el doctorando. Si no hay ninguna me provoca automáticamente una cierta prevención sobre su calidad. Publicar si se quiere desarrollar una carrera investigadora es lo más importante. Y llegar a doctorarse sin haber escrito nada sobre el tema de la tesis no es la mejor carta de presentación; lógicamente he encontrado alguna excepción.

No contamos con estudios al respecto en arqueología pero sí algún trabajo pionero (Hatch y Skipper 2016) sobre otras disciplinas sociales que indica que los doctorandos ya publican, antes de la presentación de la tesis, una media de algo más de cuatro artículos o capítulos de libro. Esto es una buena práctica en absoluto criticable; es más, debe formar parte fundamental de la formación del doctorado (Stoicescu y Douglas 2010). El conflicto viene de la presión por publicar, mucho y en revistas de la mayor calidad posible, porque entonces surgen dos problemas: por un lado, la mala preparación de los doctorandos para producir artículos en revistas de prestigio que hace difícil que tengan éxito y, en otro extremo, la "intertextualidad propia", es decir la propuesta de un texto, con pequeñas — a veces mínimas- variaciones respecto a otro ya publicado. O si quiere el fenómeno de la (re)publicación, que se justifica con múltiples argumentaciones: "se trata de otro enfoque", "hay algún dato nuevo", "va dirigido a otra audiencia" y cosas similares. Las revistas ven cada vez más difícil tratar con este tema crucial: publicar investigación verdaderamente original, a pesar de la exigencia obvia de recibir trabajos inéditos y no remitidos a otras instituciones. Indudablemente la fuerte presión del "publica o perece" hace complicado encontrar fórmulas para combatir el problema.

\section{La importancia de la/s lengua/s}

La comunicación académica funciona básicamente a través de congresos, reuniones y revistas. El idioma en que publicamos es obvio que condiciona el impacto de las publicaciones (Kristiansen 2001) y hoy el idioma internacional de la arqueología como en todas las demás ciencias, es el inglés (Gordin 2015), lo que no quiere decir, en absoluto, que la ciencia monolingüe sea deseable (Lang 2000). Porque la mainstream científica más poderosa es la anglosajona - otras como la alemana o francesa se sitúan unos puestos por detrás- y el inglés es la lingua franca de la ciencia (Neustupny 1998). Luego quedamos las minorities, las tradiciones arqueológicas con lenguas con pocos hablantes como el danés o el noruego o con muchos pero con poco peso en la investigación mundial como el castellano. Y si las minorías arqueológicas queremos tener visibilidad internacional no hay, en mi opinión, otra alternativa que o bien publicar en inglés (Harding 2007: 122-123) en revistas internacionales o bien en las nuestras incluir artículos en inglés (Mizoguchi 2015: 20). Hasta para quejarse del monolingüismo en inglés hay que hacerlo en inglés (Lang 2000) y a pesar del peso del inglés debemos reconocer la celebración de la diversidad lingüística europea porque es un valor que ha enriquecido la arqueología del continente (Willems 2008).

Tal vez podamos tener traducciones simultáneas de textos en soportes electrónicos muy pronto y así caerá la Torre de Babel (Harding 2007: 124). En cualquier caso la capacidad de leer en varios idiomas no solo tiene que ver con facilitar el acceso a datos y conocimientos de artículos de revistas, también significa desarrollar actitudes más abiertas y respetuosas con la diversidad lingüística y, no cabe duda, formas de comprensión más holísticas y profundas que simples traducciones burdas para saber de qué va un texto. La lectura de textos en otros idiomas contribuye, qué duda cabe, a conocer más profundamente las distintas tradiciones arqueológicas.

\section{La tensión papel versus electrónico}

Después de unas décadas con la penetración creciente y continua de la publicación electrónica (Boast y Biehl 2011; Samida 2006) y el anuncio de la muerte del papel el debate actual ya no es realmente entre la publicación en papel y la electrónica, sino más bien qué opción de edición electrónica elegir (Aubin 2007: 176), ya que existen distintas posibilidades (http:// www.revues.org). No sin dejar de recordar, como señalamos más arriba, que hasta ahora apenas han surgido revistas exclusivamente digitales, que la norma mayoritaria en el segmento de calidad más alta es la publicación doble (papel/electrónica) y que la gran incógnita es como nos moveremos a la edición exclusivamente electrónica, porque eso llegará. Las opciones son las siguientes: 1) edición electrónica mimética de la versión en papel; 2) puesta on line de la edición electrónica diferida, con embargo de un plazo de tiempo más o menos corto; 3) edición con complementos electrónicos que sostienen las argumentaciones científicas y no se incluyen en la versión papel; 4) publicación exclusivamente electrónica, y 5) alojamiento de los contenidos de las revistas en repositorios patrimoniales e institucionales.

En cualquier caso, que la edición digital es el presente y el futuro es una obviedad (véase un magnífico dosier en VV.AA. 2013). En ese sentido creo que solo hay dos preguntas relevantes: 1) si eso implica que serán exclusivamente digitales y cuando y 2) como se garantizará su futuro cuando los soportes electrónicos devengan obsoletos —que lo serán y además en muy breve plazo - al no ser la arqueología un campo de conocimiento con rentabilidad económica directa. Por otro lado es cierto que la digitalización es esencialmente un problema cultural porque en el fondo lo 
que pensamos es que mientras tenemos una revista entre las manos sentimos su perdurabilidad y eso no sucede con el formato electrónico (Gil 2016: 107). En el fondo estoy de acuerdo completamente en que "oponerse, levantar barreras, generar prejuicios sobre la lectura en pantallas es finiquitar el futuro" (Gil 2016: 111). Pero eso no significa automáticamente que la cantidad y velocidad de lo que nos ofrece el mundo digital es lo mejor y, sobre todo, que su crecimiento pueda ser continuo e ilimitado. Además la consideración desde una perspectiva política, con mayúscula, y de responsabilidad social de la publicación (y el impacto más amplio de Internet) en el mundo actual es muy necesaria aunque apenas haya sido debatida (Perry y Beale 2015). La arqueología, como disciplina que se ocupa del pasado, tiene que estar firmemente comprometida con el presente en que vivimos y con el futuro por llegar (Hamilakis 2015), también en el mundo de la publicación (Perry y Beale 2015: 162).

La edición de revistas académicas de arqueología está en un momento muy interesante aunque ofrezca algunos desconciertos y no pocas incógnitas (Carver 2007b). La edición de revistas de arqueología — con la prevalencia de lo digital— está ya exigiendo un reordenamiento que solo puede estar relacionado con la forma en que hacemos ciencia, esto es producimos y consumimos contenidos científicos (Oswald 2015). Es decir los puntos calientes son: los alojamientos/ soportes físicos y digitales, la accesibilidad, el copyright y la sostenibilidad de las publicaciones. ¿Qué futuro tiene por delante la publicación en papel? Es difícil de predecir pero creo que probablemente haya que pensar en soluciones híbridas y no en la simplista confrontación papel-digital (Herr 2013). Sin ninguna duda el paso del papel al medio digital ha afectado a la propia naturaleza de los textos, y aunque lo digital tiene fórmulas muy breves también hay que recordar que permite textos muy largos y con muchas ilustraciones, no hay que ahorrar papel y no hay que encajar espacios de forma precisa. Eso se traduce en publicaciones digitales con artículos de más de cien páginas, no hay problema, bueno como les digo a mis estudiantes, sí, en realidad sí que hay un problema, y grave: ¿Quién demonios va a leer nuestro macro-artículo? Y por último, volviendo a lo de soluciones híbridas, empiezan a surgir casos de revistas "anfibias", en feliz expresión de Delgado-Gal (2015: 12): pasaron del papel a lo digital en exclusiva y de lo digital han vuelto a reaparecer en papel, manteniendo la edición electrónica.

Otra de las novedades que Internet y la publicación on-line han traído es la ampliación del propio concepto de "publicación" pues a las categorías tradicionales (monografías, libros editados, capítulos de libro, recensiones, artículos-recensión, actas de congresos, notas y artículos de revista) hay que añadir las plataformas on line: los blogs, podcasts, links, tweets y entradas en Facebook. Los formatos tradicionales han sido definidos como "macro-unidades" de publicación académica frente a esos últimos que serían las "micro-unidades" de comunicación digital (Pound 2013). Especialmente los blogs (Caraher y Reinhard 2015, Rocks-Macqueen y Webster 2014, Smith 2015) y, muy seguramente, los podcasts parece que tendrán un valor creciente (Gaillet y Guglielmo 2014: 35-49). Por otro lado, el crecimiento de esas "microunidades" es vertiginoso y la pregunta importante es ¿cómo se relacionarán las "micro-unidades" con los formatos macro? Si prevalecen las "micro-unidades" -y como piensa Pound (2013) lo pueden hacerentonces surgirá un conjunto complejo de nuevos documentos para la bibliometría y la evaluación de impacto de las publicaciones. Eso quiere decir que ¿empezaremos a citar entradas de blogs y cosas por el estilo? Personalmente ya lo hago y seguro que lo haremos más en el futuro próximo.
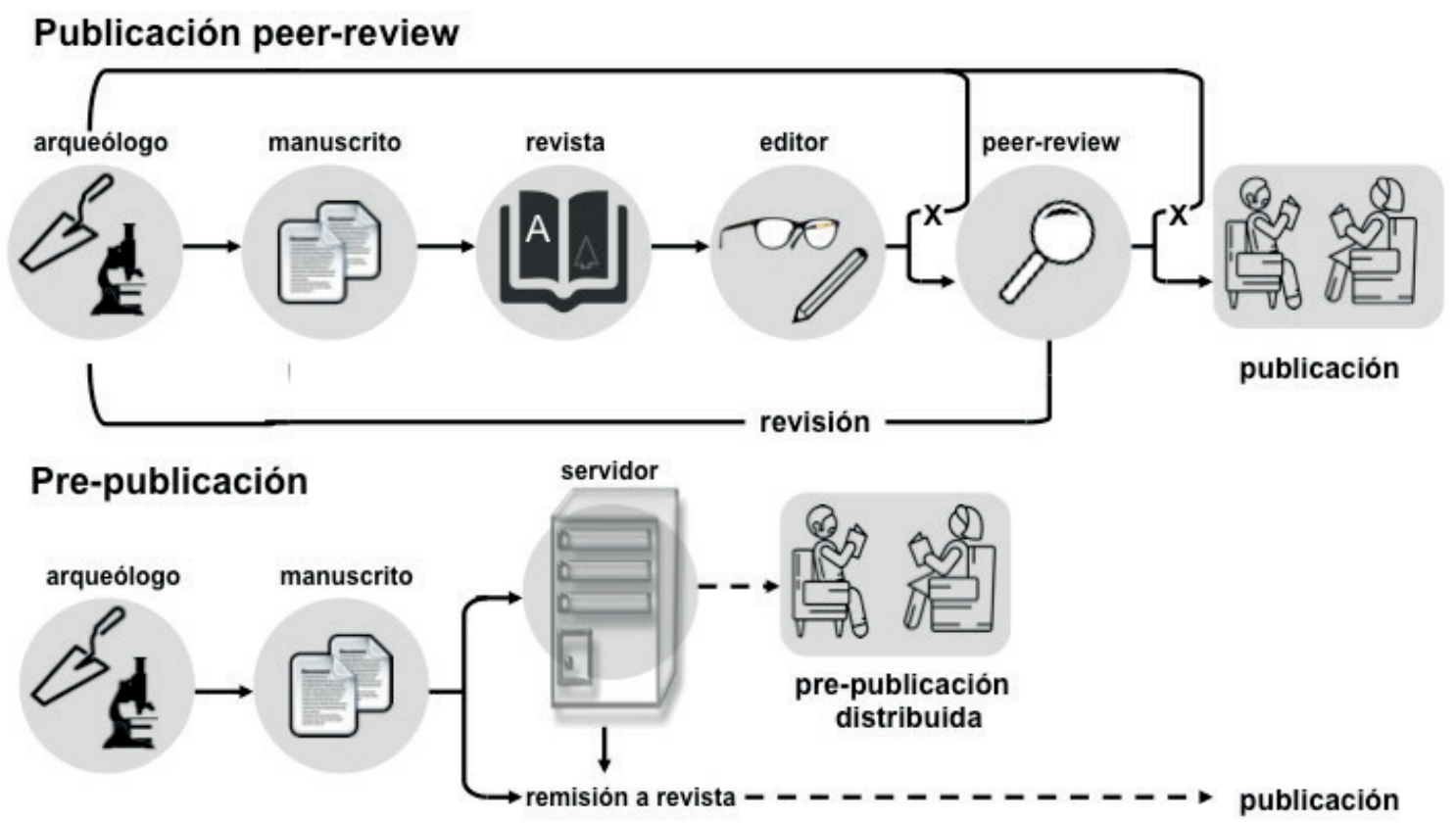

Figura 10. Comparación de los modelos de edición tradicional de evaluación por pares (peer-review) y del sistema preprint. El segundo acelera el tiempo de distribución del artículo. (Según Berg et al. 2016 modificado.) 
En cuanto a la rapidez, frente al modelo tradicional de publicación con evaluadores el sistema preprint (Berg et al. 2016) permite publicar en menos tiempo (figura 10). Un preprint es un manuscrito científico completo (a menudo también enviado a una revista con evaluación de pares) que es subido por los autores a un servidor público sin evaluación formal. Después de un tiempo de consulta por investigadores para garantizar su cualificación el manuscrito se puede ver en la Web. Así los servidores preprint facilitan el acceso directo y abierto de nuevos conocimientos a la comunidad científica mundial antes de la validación tradicional de la peer-review.

Las redes sociales académicas están remodelando aceleradamente el paisaje de la publicación científica y sus cifras son muy estimables; en 2008 Academia. edu tenía 34 millones de académicos, ResearchGate unos 9 millones de miembros y Mendeley contaba con algo más de 4,5 millones de usuarios (Matthews 2016). Estas redes proporcionan alta visibilidad, son más ágiles, cómodas y rápidas que los repositorios institucionales y además cuentan con otros servicios útiles al investigador. Considero en cambio que validar los hits de un investigador en esas redes como méritos en evaluaciones y procesos de promoción es un exceso y un síntoma más de narcisismo digital. Por último, estas redes son ya una alternativa, aunque con limitaciones, a los editores académicos tradicionales a los que, de todos modos, no aventajan en reputación y control de calidad pero sí en capacidad de difusión. En cualquier caso ya hemos visto como han afectado a la peer-review tradicional, es posible que en un futuro inmediato puedan dar el salto para convertirse en editores y la cuestión más compleja y delicada es como tratarán con el Open Access sin convertirse en mercaderes ni ofender a los académicos.

Como escribió Alan Vince (1996) en el editorial del primer número de Internet Archaeology "la verdad es que no tenemos ni idea de lo que nos espera [en el futuro] o cual será el ritmo del cambio". Y veinte años después podemos decir exactamente lo mismo aunque ya contamos con bastantes experiencias en el ámbito de la publicación digital en sus diversas modalidades para pensar un escenario todavía más cambiante y con ritmos más acelerados, pero seguramente prometedor.

\section{Prestigio, descubribilidad y visibilidad}

Aunque no tengamos cifras contrastadas de las comunidades arqueológicas del mundo, salvo algunas pocas de los países más desarrollados arqueológicamente (Aitchison 2015), es evidente que el número de arqueólogos en los últimos treinta años no ha dejado de crecer a nivel mundial (una estimación razonable podría estar entre 30.000 y 50.000), a pesar del importante impacto de la crisis económica iniciada en 2008. Con ello hemos visto el paralelo crecimiento del número de revistas y artículos publicados anualmente. La expansión del ecosistema de las revistas de arqueología lo ha hecho también más complejo. Y la presión académica para publicar se ejerce "en vertical", presionando con originales sobre las revistas percibidas como más prestigiosas, atractivas y reconocidas en los sistemas de evaluación y promoción y también "en horizontal", sobre las revistas nuevas, con menos exigencias y contribuyendo, además, a crear otras nuevas. De hecho el crecimiento de revistas testimonia una cierta "saturación" de las mejores y la fundación de nuevas no deja de ser muchas veces una espita de ampliación del mercado para publicar.

Pero un problema común para las revistas es ganar prestigio, para así obtener más propuestas de originales, poder seleccionar los mejores y así reproducir el sistema. Buenos artículos, significa probablemente tener más citas, y más citas de ciertos artículos construyen su impacto y por extensión de la revista donde fue publicado. Los rankings, a través de los Citation Indexes internacionales (Web of Science y Scopus) y los regionales, posicionan objetivamente las revistas, mediante el factor de impacto, y contribuyen al prestigio percibido por la comunidad arqueológica. Aún así tener una descubribilidad alta o de $360^{\circ}$, es decir conseguir que la revista $\mathrm{X}$ sea fácilmente encontrada en el universo del papel impreso y de la edición digital exige también esfuerzos por parte de los editores. El dominio anglosajón con la arqueología en inglés es difícil de penetrar por la barrera idiomática y la descubribilidad de las revistas en castellano es baja. En fin, la obsesión de alcanzar prestigio mensurable está haciendo que se desarrollen procedimientos, como el Search Engine Optimization (SEO) para incrementar visitas a los artículos y ayudar a tener más visitas, descargas y citas (Lotfipanah 2015).

Un problema importante para las revistas de "minorías arqueológicas", como la nuestra, es que su factor de impacto por debajo de las internacionales en inglés drena buenos trabajos nuestros hacia esas revistas, contribuyendo, en cierto modo y sin voluntad de ello, al debilitamiento de nuestras propias revistas. El crecimiento de contribuciones españolas en el Journal of Archaeological Science es, en este sentido, muy expresivo: de 7 artículos en 1985-1994, pasamos a 51 en 1995-2004 y a 272 (!) en 2005-2014, y somos el $4^{\circ}$ país, solo por detrás de EE.UU., Reino Unido y Francia (Torrence et al. 2015). Fantástica evolución en poco más de dos décadas pero también para reflexionar sobre la pérdida que, en algún sentido, representa para las revistas españolas. La inclusión de buenos artículos en inglés en nuestras revistas creo que es una de las pocas buenas alternativas para incrementar su prestigio - aunque no todos lo consideran asíy luchar por nuestro posicionamiento internacional. Defender y mejorar la calidad de nuestras revistas debe ser una obligación de todos. Será la única manera de ganar visibilidad —una mezcla especial de diseminación, accesibilidad y reconocimiento de prestigio- y llamar la atención sobre la calidad de la arqueología en nuestro país. Una buena revista contribuye muy positivamente a la construcción del patrimonio colectivo de la disciplina. Y como en el caso francés pienso que apostar por las cabeceras existentes será más inteligente que la creación de nuevas revistas.

Gonzalo Ruiz Zapatero Universidad Complutense 
Aitchison, K. (2015). Archaeological market survey 2015. Landward Research for CIFA/FAME (www. landward.eu/Archaeological_Market_Survey_2015.pdf) (acceso 10-06-2016).

Ames, K. (2014). Publishing archaeology in the twenty-first century. The SAA Archaeological Record, 14 (4): 3-4.

AnNOUnCEMENT (2015). Nature journals offer double-blind review. Nature, 518: 274.

ARns, M. (2014). Open access is tiring out peer reviewers. Nature, 515: 467.

Aubin, G. (coord.) (2007). Enquête sur les revues d'archéologie du territoire national. Comité des publications et de la diffusion de la recherche archéologique (CPDRA). París (www.culturecommunication. gouv.fr/content/.../1/.../enquete_revues_archeo_2007.pdf) (acceso 10-06-2016).

Aubin, G. (2012). Les metiers de l'archéologie. La publication scientifique. Archeótema. Revue d'histoire et d'archéologie, Hors serie 5: 96-97.

Berg, J. M. et al. (24 firmantes) (2016). Preprints for the life sciences. Science, 352: 899-901.

BoAst, R. y BieHL, P. (2011). Archaeological Knowledge Production and Dissemination in the Digital Age. En: Kansa, E. C., Kansa, S. W. y Watrall, E. (eds.). Archaeology 2.0 New approaches to communication \& Collaboration: 119-158 (http://escholarship.org/uc/ item/1r6137tb\#page-3) (acceso 11-06-2016).

Bohannon, J. (2013). Who's Afraid of Peer Review? Science, 342: 60-65.

Bruce, A. (2008). Scientific Publishing Standards. Science, 321: 1271.

Butler, D. (2013). The Dark Side of Publishing. Nature, 495: 433-435.

Canny, N. (2015). Opening Access to Archaeology. Archäologische Informationen, 38: 21-29.

Caraher, W. R. y Reinhard, A. (2015). From Blogs to Books: Blogging as Community Practice, and Platform. Internet Archaeology, 39 (http://intarch.ac.uk/journal/ issue39/7/index.html) (acceso: 8/06/2016).

CARver, M. (2007a). Archaeology journals, academics and Open Access. European Journal of Archaeology, 10 (2-3): 135-148.

Carver, M. (2007b). The future of Antiquity. En: M. RundKVIST (ed.). Scholarly journals between the past and the future: the Fornvännen centenary round-table seminar, Stockholmm 21 April 2006 (Konferenser 65)
Kungl. Vitterhets Historie och Antikvitets Akademien. Stockholm: 30-49.

Casati, R. (2015). Elogio del papel. Contra el colonialismo digital. Ariel. Barcelona.

Connah, G. (2010). Writing about archaeology. Cambridge University Press. Cambridge.

Costopoulos, A. (2016). Digital Archeology Is Here (and Has Been for a While). Frontiers in Digital Humanities / Digital Archaeology (http://journal.frontiersin. org/article/10.3389/fdigh.2016.00004/full).

Chippindale, C. (2005). Colleagues, talking, writing, publishing. En: H. D. G. Maschner y C. Chippindale (eds.). Handbook of Archaeological Methods. Altamira Press, II. Lanham: 1339-1371.

Delgado-Gal, A. (2015). Editorial. Revista de Libros, 181: 11-12 (http://www.revistadelibros.com/descargas/ RdL181-revista-de-libros.pdf) (acceso 11-06-2016).

Donovan, S. K. (2014). How to Be an Effective Peer Reviewer. Journal of Scholarly Publishing, 46 (1): 89-95.

Editorial (2015). Publish or perish. Nature, 521: 259.

Ferguson, C., Marcus, A. y Oransky, I. (2014). The Peer-Review Scam. Nature, 515: 480-482.

FitzPatrick, K. (2011). Planned Obsolescence: Publishing, Technology, and the Future of the Academy. New York University Press. New York.

Frame, L. y Duwe, F. (2009). The where's, why's, and how's of student publishing. The SAA Archaeological record, 9 (4): 36-37.

Gaillet, L. L. y Guglielmo, M. (2014). Scholarly Publication in a Changing Academic Landscape: Models for Success. Palgrave Macmillan. Nueva York.

Gero, J. y Root, D. (1990). Presentations and Private concerns: Archaeology in the pages of National Geographic. En: Gathercole, P. y Lowenthal, D. (eds.). The Politics of the Past. Unwin Hyman. Londres: 19-37.

GIL, M. (2016). Repensar el ecosistema del libro. Texturas, 29: 107-111.

Gordin, M. (2015). Scientific Babel: The Language of Science from the Fall of Latin to the Rise of English. University Chicago Press. Chicago.

Hamilakis, Y. (2015). Archaeology and the Logic of Capital: Pulling the Emergency Brake. International Journal of Historical Archaeology, 19 (4): 721-735.

HaRding, A. (2007). Communication in Archaeology. European Journal of Archaeology, 10 (2-3): 119-133. 
Harding, A. (2015). Editorial. Open Archaeology, 1 (1) (http://www.degruyter.com/view/j/opar.2014.1.issue-1/ issue-files/opar.2014.1.issue-1.xml) (acceso 11-06-2016).

Hatch, T. y Skipper, A. (2016). How Much Are Students Publishing before Graduation?: An Examination of Four Social Science Disciplines. Journal of Scholarly Publishing, 47 (2): 171-179.

Herr, S. (2013). A Future of Archaeological Publishing. The SAA Archaeological Record, 13 (1): 8-13.

Hirsch, J. E. (2005). An index to quantify an individual's scientific research output. PNAS, 102, 46: 16569-16572.

Joyce, R. A., Guyer, C., Joyce, M., Lopiparo, J. y PreuCEL, R. (2002). The languages of archaeology: Dialogues, narrative and writing. Blackwell. Oxford.

Kohle, H. (2015). Publish first - filter later. Über den Prozess der Qualitätsbewertung im Open Access. Archäologische Informationen, 38: 109-112.

Котв, Е. (2016). How do journals select competent reviewers when peer reviewing? (https://www.researchgate.net/post/How_do_journals_select_competent_reviewers_when_peer_reviewing) (acceso 11-06-2016).

Kristiansen, K. (2001). Borders of Ignorance: Research Communities and Language. En: KobYLinski, Z. (ed.). Quo Vadis Archaeologia? Whither European Archaeology in the 21st Century. European Science Foundation/ Polish Academy of Sciences. Varsovia: 38-43.

LANG, V. (2000). Archaeology and language. Fennoscandia archaeologica, XVI (I): 103-110.

LaVell, C. (1981). Publication: an obligation. Archaeological documentation in Britain today. Bulletin of the Institute of Archaeology, University of London, 18: 91-125.

Lavivière, V., Haustein, S. y Mongeon, Ph. (2015). The oligopoly of Academic Publishers in the Digital Era. PLOS ONE, 10 (6) (http://journals.plos.org/plosone/ article?id=10.1371/journal.pone.0127502) (acceso 1106-2016).

Linares Matás, G. (2016). A Student Perspective on the Present of Archaeology: JSRA Editorial. International Journal of Student Research in Archaeology (IJSRA), 1 (1): i-vii.

Lipscombe, T. (2016). Burn This Article: An Inflammatory View of Peer Review. Journal of Scholarly Publishing, 47 (3): 284-298.

Lotfipanah, M. (2015). Does search engine optimisation (SEO) increase scientific journals' visibility, prestige, and impact factor as a new method? (https://www. researchgate.net/publication/285871747_Does_SEO_increase_scientific_journals_visibility_prestige_and_impact_factor_as_a_new_method) (acceso 14-06-2016).

Lovejoy, T. I., Revenson, T. A., France, Ch. R. (2011). Reviewing Manuscripts for Peer-Review Journals: A Primer for Novice and Seasonal Reviewers. Annals of Behavioral Medicine, 42: 1-13.
Lucas, G. (2001). Destruction and the rhetoric of excavation. Norwegian Archaeological Review, 34 (1): 35-46.

McManamon, F. P. y Kintigh, K. W. (2010). Digital Antiquity: Transforming Archaeological Data into Knowledge. The SAA Archaeological Record, 10 (2): 37-40.

Martínez Navarrete, M. I., Montero Ruiz, I. (2004). Editorial. Calidad de revistas científicas. Trabajos de Prehistoria, 61 (2): 5-7.

Matthews, D. (2016). Do academic social networks share academics' interests? Times Higher Education, 7 April 2016 (https://www.timeshighereducation.com/ features/do-academic-social-networks-share-academics-interests).

Meruane, O. S., González Vergara, C. y Pina-Stranger, A. (2016). What We Still Don't Know About Peer Review. Journal of Scholarly Publishing, 47 (2): 187-212.

Mizoguchi, K. (2015). A Future of Archaeology. Antiquity, 89 (343): 12-22.

Mora Rodríguez, G. (1995). La arqueología en las revistas de arte del siglo xIX. Historiografía del arte español en los siglos XIX y XX. VII Jornadas de Arte (22-25 noviembre de 1994): 161-170.

Mora Rodríguez, G. (2002). Archivo Español de Arqueología: notas para una historia de la revista. Archivo Español de Arqueología, 75 (186-86): 5-20.

Mora Rodríguez, G. y Ramírez, M. (en prensa). La institucionalización científica de la Arqueología en España a través de sus revistas y series periódicas. En: $I V$ Congreso Internacional de Historia de la Arqueología y III Jornadas de Historiografía de la Arqueología SEHAMAN: 150 años de arqueología: teoría y método de una disciplina (11-13 diciembre 2014). Museo Arqueológico Nacional. Madrid.

Morgan, C. y Eve, S. (2012). DIY and digital archaeology: what are you doing to participate? World Archaeology, 44: 521-537.

Neustupny, E. (1998). Mainstreams and Minorities in Archaeology. Archaeologia Polona, 35-36: 13-23.

Oswald, K. (2015). Hand in Hand. Forschung und Vermittlung in der digitalen Archäologie. Archäologische Informationen, 38 (http://www.dguf.de/fileadmin/AI/ ArchInf-EV_Oswald.pdf) (acceso 14-06-2016).

Perkel, J. M. (2015). Rate that Journal. Nature, 520: 119-120.

Perry, S. y Beale, N. (2015). The Social Web and Archaeology's Restructuring: Impact, Exploitation, Disciplinary Change. Open Archaeology, 1 (1): 153-165.

Pinholster, G. (2016). Journals and funders confront implicit bias in peer review. Science, 352 (6289): 1067-1068. 
Pound, S. (2013). Amodern 1: the future of the scholarly journal (http://amodern.net/article/the-future-ofthe-scholarly-journal/) (acceso 13-06-2016).

Price, D. J. DE Solla (1978). Editorial statement. Scientometrics, 1 (1): 7-8.

RICHARDSON, L.-J. (2014). Understanding Archaeological Authority in a Digital Context. Internet Archaeology, 38 (http://intarch.ac.uk/journal/issue38/richardson_index. html) (acceso 14-06-2016).

Rocks-Macoueen, D. (2015a). May you live in interesting times - Publishing and Open Access in Archaeology. Archäologische Informationen, 38: 41-52.

Rocks-Macoueen, D. (2015b). Doug's Archaeology Blog (http://dougsarchaeology.wordpress.com/) (acceso 1006-2016).

Rocks-Macoueen, D. y Webster Ch. (eds.) (2014). Blogging Archaeology. Landwall Research Ltd in association with Succint Research and DIGTECH LLC (http://www.digtech-llc.com/blogarch-ebook/) (acceso 10-06-2016).

RundKvist, M. (2007). Scholarly Journals between the Past and the Future: The Fornvännen Centenary RoundTable Seminar, Stockholm 21 April 2006. Konferenser 65. Vitterhets Historie och Antikvitets Akademien. Kungl (https://alunsalt.com/scholarly-journals-betweenthe-past-and-the-future-by-martin-rundkvist-b90d7bb5cd36\#.kmvos73qn) (acceso 13-06-2016).

Ruiz Zapatero, G. (1987). La recensión de publicaciones arqueológicas: S.O.S. Trabajos de Prehistoria, 44: 313-321.

Ruiz Zapatero, G. (1997). Las "caras ocultas" de la crítica arqueológica. Revista d'Arqueologia de Ponent, 7: 274-277.

Ruiz Zapatero, G. y Álvarez Sanchís, J. R. (1989). Arqueología y publicación. Revista de Arqueología, 96: 5-11.

SAmIDA, S. (2006). Elektronische Zeitschriften in der Ur- und Frühgeschichtlichen Archäologie: Bestandsaufnahme und Analyse. Bibliotheksdienst, 40: 1003-1014.

SAREwitz, D. (2016). The pressure to publish pushes down quality. Nature, 533: 147.

Schniermeir, Q. (2016). Peer-review: close inspection. Nature, 533: 279-282.

SIDERIS, A. (2004). Scientific archaeological publications in the Digital Era (https://www.academia.edu/481236/ Scientific_Archaeological_Publications_in_the_Digital_Era) (acceso: 11-06-2016).

Sмiтн, M. (2015). Publishing Archaeology Blog (http:// publishingarchaeology.blogspot.co.uk/[1.3.2015]) (acceso: 10-06-2016).
Stoicescu, D. y Douglas, M. (2010). Starting to Publish Academic Research as a Doctoral Student. International Journal of Doctoral Studies, 5: 79-92.

Swogger, J. G. (2015). Ceramics, Polity \& Comics: Visually Representing Formal Archaeological Text. Advances in Archaeological Practice, 3 (1): 16-28 (https:// johngswogger.wordpress.com/2015/02/23/comics-andacademia/) (acceso 30-05-2016).

SzuTER, C. (2014). Publishing archaeology in the twentyfirst century: archaeological values, hidden publishing costs, public engagement, and data prestige. The $S A A$ Archaeological Record, 14 (4): 4-5.

Taylor\&Francis Group (2015). Peer review in 2015. A global view (http://authorservices.taylorandfrancis.com/ wp-content/uploads/2015/10/Peer-Review-2015-whitepaper.pdf) (acceso 12-06-2016).

Torrence, R., Martinon-Torres, M., Rheren, T. (2015). Forty years and still growing: Journal of Archaeological Science looks to the future. Journal of Archaeological Science, 56: 1-8.

VINCE, A. (1996). Editorial. Internet Archaeology, 1 (http://intarch.ac.uk/journal/issue1/edit1.html) (acceso 10-06-2016).

Vora, N. y Boellstorff, T. (2012). Anatomy of an article: the peer-review process as method. American Anthropologist, 114(4): 578-583.

VV.AA. (1986). La publication en archéologie. Mélanges de l'Ecole Française de Rome, Antiquités, 98 (1): 359-386.

VV.AA. (2013). Communication in Science. Pressures and Predators. Science, 342: 13-82.

Wattham, M. (2009). The Future of Scholarly Journals Publishing among Social Science and Humanities Associations. Report on a study funded by a planning grant from the Andrew W. Mellon Foundation (www. nhalliance.org/bm doc/hssreport.pdf).

WERNER, R. (2015). The focus in bibliometrics makes papers less useful. Nature, 517: 245.

Willems, W. J. H. (2008). Language and Archaeology. Archaeologies: Journal of the World Archaeological Congress, 4 (1): 179-181.

WiLSDon, J. (2015). We need a measured approach to metrics. Nature, 523: 129.

Woolston, C. (2015). Build a reputation. Nature, 521: 113-115.

XIA, J. (2006). Electronic Publishing in Archaeology. Journal of Scholarly Publishing, 37(4): 270-287.

XIA, J. (2011). Open Access for Archaeological Literature: A Manager's Perspective. En: E. C. KanSA, S. Whitcher Kansa \& E. Watrall (eds.). Archaeology 2.0. New Approaches to Communication and Collaboration. Cotsen Institute of Archaeology Press. Los Angeles: 233-249. 